\title{
Epac2-deficiency leads to more severe retinal swelling, glial reactivity and oxidative stress in transient middle cerebral artery occlusion induced ischemic retinopathy
}

\author{
LIU Jin ${ }^{1}$, YEUNG Patrick Ka Kit ${ }^{1}$, CHENG Lu ${ }^{1}$, LO Amy Cheuk Yin ${ }^{2,3}$, \\ CHUNG Stephen Sum Man ${ }^{4} \&$ CHUNG Sookja Kim ${ }^{1,3,5^{*}}$ \\ ${ }^{1}$ Department of Anatomy, Li Ka Shing Faculty of Medicine, The University of Hong Kong, Hong Kong, China; \\ ${ }^{2}$ Department of Ophthalmology, Li Ka Shing Faculty of Medicine, The University of Hong Kong, Hong Kong, China; \\ ${ }^{3}$ Research Centre of Heart, Brain, Hormone and Healthy Aging, Li Ka Shing Faculty of Medicine, The University of Hong Kong, Hong Kong, \\ China; \\ ${ }^{4}$ Division of Science and Technology, United International College, Zhuhai 519085, China \\ ${ }^{5}$ State Key Laboratory of Pharmaceutical Biotechnology, The University of Hong Kong, Hong Kong, China
}

Received December 12, 2014; accepted March 13, 2015; published online May 18, 2015

\begin{abstract}
Ischemia occurs in diabetic retinopathy with neuronal loss, edema, glial cell reactivity and oxidative stress. Epacs, consisting of Epac1 and Epac2, are cAMP mediators playing important roles in maintenance of endothelial barrier and neuronal functions. To investigate the roles of Epacs in the pathogenesis of ischemic retinopathy, transient middle cerebral artery occlusion (tMCAO) was performed on Epacl-deficient $\left(E p a c 1^{--}\right)$mice, Epac2-deficient $\left(E p a c 2^{-/}\right)$mice, and their wild type counterparts $\left(\right.$ Epacl $^{+/+}$and $\left.E \mathrm{Fac2}^{+/+}\right)$. Two-hour occlusion and 22-hour reperfusion were conducted to induce ischemia/reperfusion injury to the retina. After tMCAO, the contralateral retinae displayed similar morphology between different genotypes. Neuronal loss, retinal edema and increase in immunoreactivity for aquaporin 4 (AQP4), glial fibrillary acidic protein (GFAP), peroxiredoxin 6 (Prx6) were observed in ipsilateral retinae. Epac2 ${ }^{-/-}$ipsilateral retinae showed more neuronal loss in retinal ganglion cell layer, increased retinal thickness and stronger immunostaining of AQP4, GFAP, and Prx6 than those of Epac2 $2^{+/+}$. However, Epacl ${ }^{-/-}$ipsilateral retinae displayed similar pathology as those in Epacl $^{+/+}$mice. Our observations suggest that Epac2-deficiency led to more severe ischemic retinopathy after retinal ischemia/reperfusion injury.
\end{abstract}

Epac, retina, ischemia, retinopathy

Citation: $\quad$ Liu J, Yeung PKK, Cheng L, Lo ACY, Chung SSM, Chung SK. Epac2-deficiency leads to more severe retinal swelling, glial reactivity and oxidative stress in transient middle cerebral artery occlusion induced ischemic retinopathy. Sci China Life Sci, 2015, 58: 521-530, doi: $10.1007 / \mathrm{s} 11427-015-4860-1$

Ischemia occurs in several retinal diseases, including diabetic retinopathy, glaucoma, and central retinal artery occlusion [1]. Dysfunction of retinal capillary is the major cause of retinal ischemia. Deprivation of blood supply induces a series of pathophysiological changes in the retina. Neuronal cell death in the retinal ganglion cell layer (GCL) is observed as pyknotic nuclei in the GCL [2-5]. Glial acti-

*Corresponding author (email: skchung@hkucc.hku.hk) vation is evident by up-regulation of glial fibrillary acidic protein (GFAP) in response to deleterious release of glutamate from over excited neurons after ischemia [6,7]. Retinal edema also occurs, because of the increased water transportation and accumulation as a result of increased extracellular fluid volume, increased aquaporin 4 (AQP4) immunoreactivity and swelling of retinal glial cells [8,9]. Oxidative stress is present under ischemic condition, which further exacerbates the progression of retinopathy $[10,11]$. 
Exchange proteins directly activated by cAMP (Epacs), consisting of Epac1 and Epac2, are cAMP mediators independent of protein kinase A (PKA). As a mediator of cAMP, an important second messenger for intracellular signal transduction, Epacs take part in many biological functions (reviewed in [12]). Epac1 is involved in the maintenance of cell-cell junctions [13]. The activation of Epac1 reduces the permeability of endothelial cells in vitro [14]. Epac2 participates in the modulation of neuronal activities, including neurotransmitter release and synaptic plasticity [15-17]. Both Epac1 and Epac2 are expressed in the brain [18], and retina [19], which is part of central nervous system (CNS) and can be viewed using non-invasive methods.

Therefore, we determine the molecular significance of Epac in retinal neurons and in the pathogenesis of ischemic retinopathy using the Epacl-deficient $\left(E_{p a c l}^{-/}\right)$and Epac2-deficient $\left(\right.$ Epac $\left.^{-/-}\right)$mice under transient ischemic retinopathy condition. Here, we report that, after transient middle cerebral artery occlusion (tMCAO), more severe ischemic retinopathy was observed in the ipsilateral retinae of $\mathrm{Epac}^{-/-}$mice when compared with that of Epac2 ${ }^{+/+}$mice, whereas there was no obvious difference in retinae of Epacl $^{-/-}$and Epacl $^{+/+}$mice.

\section{Materials and methods}

\subsection{Animals}

All animals used in this study were housed in a temperature-controlled room with diurnal light conditions and allowed access to food and water freely in the Laboratory Animal Unit of The University of Hong Kong.

Epacl $^{-/-}$mice were generated previously [20], and they were backcrossed to $\mathrm{C} 57 \mathrm{BL} / 6 \mathrm{~N}$ mice for more than 10 generations. Age-matched $\mathrm{Epacl}^{+/+}$littermates served as the control group. Epac2 ${ }^{-/}$mice were backcrossed to C57BL/6N mice for more than 10 generations (a kind gift from Prof. S. Seino of Kobe University); thus they were considered congenic with C57BL/6N mice. C57BL/6N mice were therefore used as the control group $\left(\right.$ Epac $\left.^{+/+}\right)$for Epac2 $^{-/-}$mice as in previous publication [21]. Three to six animals were included in each group.

\subsection{Genotyping of Epac1- and Epac2-deficient mice}

Genomic DNA was extracted from tail biopsy of each mouse. Polymerase chain reaction (PCR) was used to identify the genotype of each mouse. The sequences of primers used are as follows: Epacl (forward) 5'-GTTCTGCTCTTTGAACCACACAGCAA-3', Epacl (reverse1) 5'-AGAACTCAATCACAGCCTGTCCCTACC-3', and Epac1 (reverse2) 5'-ATCAGCAGCCTCTGTTCCAC-3', for Epac1 genotyping; Epac2 (forward1) 5'-TGTGATAAACATTCTCGATT3', Epac2 (forward2) 5'-GCATACATTATACGAAGT-
TATC-3', and Epac2 (reverse) 5'-CTGATCACATTAGCAAGCTC-3' for Epac2 genotyping. The genotyping was performed in a $50 \mu \mathrm{L}$ PCR reaction system, and the annealing temperatures were $58^{\circ} \mathrm{C}$ and $51^{\circ} \mathrm{C}$ for Epacl and Epac2, respectively. The PCR products were loaded to a $1.5 \%$ agarose gel with ethidium bromide for electrophoresis. The bands for PCR products were visualized under ultraviolet light. For Epacl genotyping, bands with two sizes could be seen: the upper $360 \mathrm{bp}$ band indicated $\mathrm{Epacl}^{+/+}$, and the lower 260 bp band was for Epacl ${ }^{-/}$; for Epac2 genotyping, the upper 211 bp band represented Epac2 ${ }^{+/+}$, whereas the lower 90 bp band was for Epac2 $2^{-/}$(Figure 1A).

\subsection{Transient middle cerebral artery occlusion (tMCAO)}

tMCAO was performed as previously described [11,22,23]. Briefly, after the mice (8-12 weeks old) were anesthetized (2\% halothane in $70 \% \mathrm{~N}_{2} \mathrm{O} / 30 \% \mathrm{O}_{2}$ for induction and $1 \%$ halothane in $70 \% \mathrm{~N}_{2} \mathrm{O} / 30 \% \mathrm{O}_{2}$ for maintenance), a nylon filament was inserted into the common carotid artery, directed to the right internal carotid artery, and finally reached the bifurcation between the middle and anterior cerebral arteries. This blocked the middle cerebral artery and its branch, the ophthalmic artery, leading to an interruption in the blood supply to the eye. Laser Doppler flowmetry (Perimed, Jarfalla, Sweden) was used to measure the relative cerebral blood flow (rCBF) of middle cerebral artery territory so as to ensure the successful occlusion and reperfusion of the middle cerebral artery. The filament was left for $2 \mathrm{~h}$ and was pulled out afterwards for a 22-h reperfusion after which the mice were sacrificed for sample collection. The ischemia was introduced to right eye (ipsilateral side) while the left eye served as a contralateral control side.

\subsection{Tissue processing}

The eyeball was enucleated $24 \mathrm{~h}$ after induction of ischemia. The cornea and lens were removed; the eye cup was fixed in $4 \%$ paraformaldehyde at room temperature for $2 \mathrm{~h}$. After dehydration with graded series of ethanol, the eye cup was embedded in paraffin wax. Seven micron-thick sections were cut for morphological analysis.

\subsection{Morphometric analysis}

The thickness of the retina layers $300 \mu \mathrm{m}$ adjacent to the optic nerve was measured in paraffin embedded retinal sections stained with hematoxylin and eosin (H\&E). The microphotographs were taken under $20 \times$ objective of an Olympus IX71 microscope system (Olympus, Japan) linked with a digital camera (SPOT CCD Digital Camera, Diagnostic Instruments Inc., Sterling Heights, MI, USA). The retinal layers being assessed were as follows: (a) outer lim- 

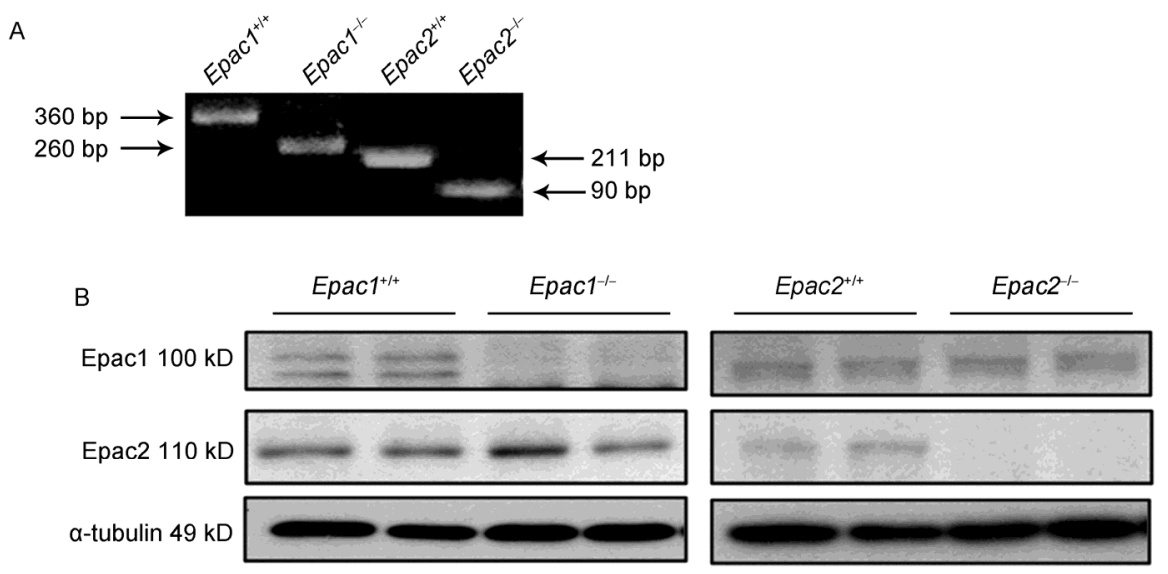

Figure 1 Confirmation of gene knockout in knockout mice. A, genotyping for $E p a c 1^{-/}$and $E p a c 2^{-/}$mice. Epacl ${ }^{+/+}$mice showed a 360 bp band, and

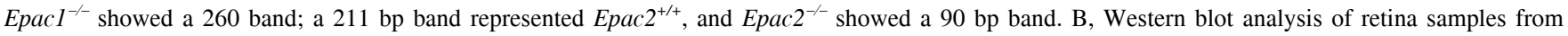
$\mathrm{Epacl}^{+/+}, \mathrm{Epacl}^{-/}, \mathrm{Epac2}^{+/+}$, and $\mathrm{Epac2}^{-/}$mice. Epac1 protein was displayed as a $100 \mathrm{kD}$ double band, whereas Epac2 protein was shown as a $110 \mathrm{kD}$ single band. No Epac1 bands were detected in $\mathrm{Epacl}^{-/-}$mice, and no Epac2 bands were detected in Epac2 ${ }^{-/}$mice.

iting membrane (OLM) to inner limiting membrane (ILM), (b) inner nuclear layer (INL), (c) inner plexiform layer (IPL), (d) outer plexiform layer (OPL), (e) outer nuclear layer (ONL), and (f) IPL to ILM. The relative differences between contralateral and ipsilateral sides in Epac-wildtype and Epac-deficient mice were compared in a blind fashion.

Cells in the GCL with pyknotic nuclei were defined as dead cells. The number of the dead cells in the GCL layer was counted in only one of the four consecutive sections of one slide to avoid double counting. The differences between contralateral and ipsilateral sides in Epac-wildtype and Epac-deficient mice were compared.

\subsection{Immunocytochemistry}

The sections were deparaffinized and rehydrated with graded series of ethanol. The sections were then immersed in $0.3 \% \mathrm{H}_{2} \mathrm{O}_{2}$ to block endogenous peroxidase, after which they were incubated with $1.5 \%$ normal goat serum for $1 \mathrm{~h}$ at room temperature. This was followed by incubation with primary antibodies overnight at $4{ }^{\circ} \mathrm{C}$. The primary antibodies used and their concentrations were as follows: rabbit anti-GFAP (1:2,000; Dako, Carpinteria, CA, USA), rabbit anti-AQP 4 (1:500; Millipore, Billerica, MA, USA), and rabbit anti-peroxiredoxin 6 (Prx6; 1:2,000; Abcam, Cambridge, MA, USA). The sections were subsequently incubated with biotinylated goat anti-rabbit secondary antibody (Vector Laboratories, Berlingame, CA, USA) for $30 \mathrm{~min}$ at room temperature. The immunoreactive signals were amplified by incubation with the avidin-biotin peroxidase complex (Vector Laboratories, Burlingame, CA, USA) for 30 min and visualized by diaminobenzidine tetrahydrochloride (Zymed Laboratory, San Francisco, CA, USA) reaction for 2 min. Finally, the sections were counterstained with hematoxylin, dehydrated, cover-slipped, and mounted with mounting medium (Permount, Fisher Scientific, Waltham,
MA, USA). Photos of the sections were taken under Olympus IX71 microscope system (Olympus) with $10 \times$ to $40 \times$ objectives linked with a digital camera (SPOT CCD Digital Camera, Diagnostic Instruments Inc.). Semiquantitative analysis was used to assess the immunoreactivity as previously described with modifications [24,25]. Briefly, all retinal sections for analysis were processed at the same time in a single round of the immunocytochemistry (ICC) experiment. After the immunocytochemical procedures, microscopic slides were randomly coded and examined in a blinded approach. Scores were given based on the intensity and location of the staining along the whole retina, a score of 1 was assigned for the weakest immunoreactivity, and a score of 5 was assigned for the strongest immunoreactivity. Retinal sections were then decoded, and the scores were compared among the experimental groups.

\subsection{Western blot analysis}

Retinae were dissected from the enucleated eyeballs and homogenized in ice-cold radio immunoprecipitation assay buffer (RIPA, $50 \mathrm{mmol} \mathrm{L}^{-1}$ Tris, $150 \mathrm{mmol} \mathrm{L}^{-1} \mathrm{NaCl}, 0.1 \%$ SDS, $0.5 \%$ sodium deoxycholate, and $1 \%$ NP-40) with freshly added protease inhibitor. The samples were kept on ice for $30 \mathrm{~min}$ and then centrifuged at 13,200 $\mathrm{r} \mathrm{min}^{-1}$ for 30 $\min$ at $4{ }^{\circ} \mathrm{C}$. The supernatant was kept as total protein lysate and quantified by Bio-Rad protein assay (Bio-Rad Laboratories Inc., Hercules, CA, USA). Proteins were separated by SDS-PAGE and then transferred to a PVDF membrane. After blocking with 5\% non-fat milk in tris-buffered saline with $0.1 \%$ Tween 20 for $1 \mathrm{~h}$ at room temperature, the membrane was then incubated with mouse anti-Epac1 $(1: 1,000)$, mouse anti-Epac2 $(1: 1,000)$ antibody, a kind gift from Dr. Johannes Bos (Utrecht University), or mouse anti- $\alpha$-tubulin (1:4,000; Sigma, St. Louis, MO, USA), at $4^{\circ} \mathrm{C}$ overnight. Proper horseradish peroxide conjugated secondary antibod- 
ies were used to probe the primary antibodies in the following day, and the protein bands were visualized using Enhanced Chemiluminescence Reagent (GE Healthcare, Buckinghamshire, UK).

\subsection{Statistical analysis}

All the data were expressed as mean \pm SEM. Statistical analyses were performed using two-way analysis of variations (ANOVA) followed by Bonferroni post-test (GraphPad Prism software, San Diego, CA, USA). Difference with $P<0.05$ was considered as statistically significant.

\section{Results}

\subsection{Confirmation of Epac deficiency}

Western blot analysis showed that Epac1 bands could be seen in $\mathrm{Epacl}^{+/+}, \mathrm{Epac2}^{+/+}$and $\mathrm{Epac2}^{-/-}$retinal samples, but not in Epacl $^{-/-}$retinal samples; Epac2 bands could be seen in $\mathrm{Epacl}^{+/+}, \mathrm{EpaCl}^{-/-}$, and Epac2 ${ }^{+/+}$samples, but no bands were seen in Epac2 ${ }^{-/-}$samples. The absence of Epac1 protein in $\mathrm{Epacl}^{-/-}$mice retinae and the absence of Epac2 protein in $\mathrm{Epac2}^{-/-}$mice retinae demonstrated that the Epac gene was successfully knocked out in the retinae of $\mathrm{Epacl}^{-/-}$and $\mathrm{Epac2}^{-/-}$mice, respectively (Figure 1B).

\subsection{Neuronal cell death after tMCAO}

In $\mathrm{Epacl}^{+/+}$and $\mathrm{Epacl}^{-/-}$mice, contralateral retinae displayed normal morphology after tMCAO (Figure 2A and C), whereas in ipsilateral retinae, lesions in GCL, IPL and INL were obvious (Figure 2B and D). Increased number of pyknotic cells was observed in ipsilateral retinal GCL after tMCAO $\left(67.4 \% \pm 1.6 \%\right.$ in $\mathrm{Epacl}^{+/+}$and $65.4 \% \pm 4.7 \%$ in Epacl $^{--}$), when compared with their contralateral side retinae $\left(9.2 \% \pm 2.5 \%\right.$ in $\mathrm{Epacl}^{+/+}$and $12.4 \% \pm 1.8 \%$ in $\mathrm{Epacl}^{-/-}$). However, there was no significant difference between these two genotypes (Figure 2E; effect of tMCAO: $F_{1,14}=309.57$, $P<0.0001$; effect of genotype: $F_{1,14}=0.03, P=0.86$; effect of interaction: $F_{1,14}=0.68, P=0.42$ ).

Between $\mathrm{Epac}^{+/+}$and $\mathrm{Epac2}^{-/-}$groups, there was no significant differences in the contralateral retinae (Figure $2 \mathrm{~F}$ and $\mathrm{H}$ ). As expected, tMCAO induced neuronal death in ipsilateral retinae $\left(57.1 \% \pm 2.9 \%\right.$ in $\mathrm{Epac}^{+/+}$and $68.9 \% \pm$ $1.2 \%$ in $\mathrm{Epac}^{-2^{-}}$), whereas no such cell death was observed in contralateral retinae $\left(4.1 \% \pm 0.1 \%\right.$ in $\mathrm{Epac}^{+/+}$and $5.1 \% \pm$ $0.8 \%$ in $\mathrm{Epac2}^{-{ }^{--}}$). Moreover, in Epac2 $2^{-/-}$group, the number of pyknotic cells observed in ipsilateral retinal GCL was significantly higher than that in $\mathrm{Epac2}^{+/+}$group mice (Figure 2J; effect of tMCAO: $F_{1,16}=3248.42, P<0.0001$; effect of genotype: $F_{1,16}=44.8, P<0.0001$; effect of interaction: $\left.F_{1,16}=37.82, P<0.0001\right)$.

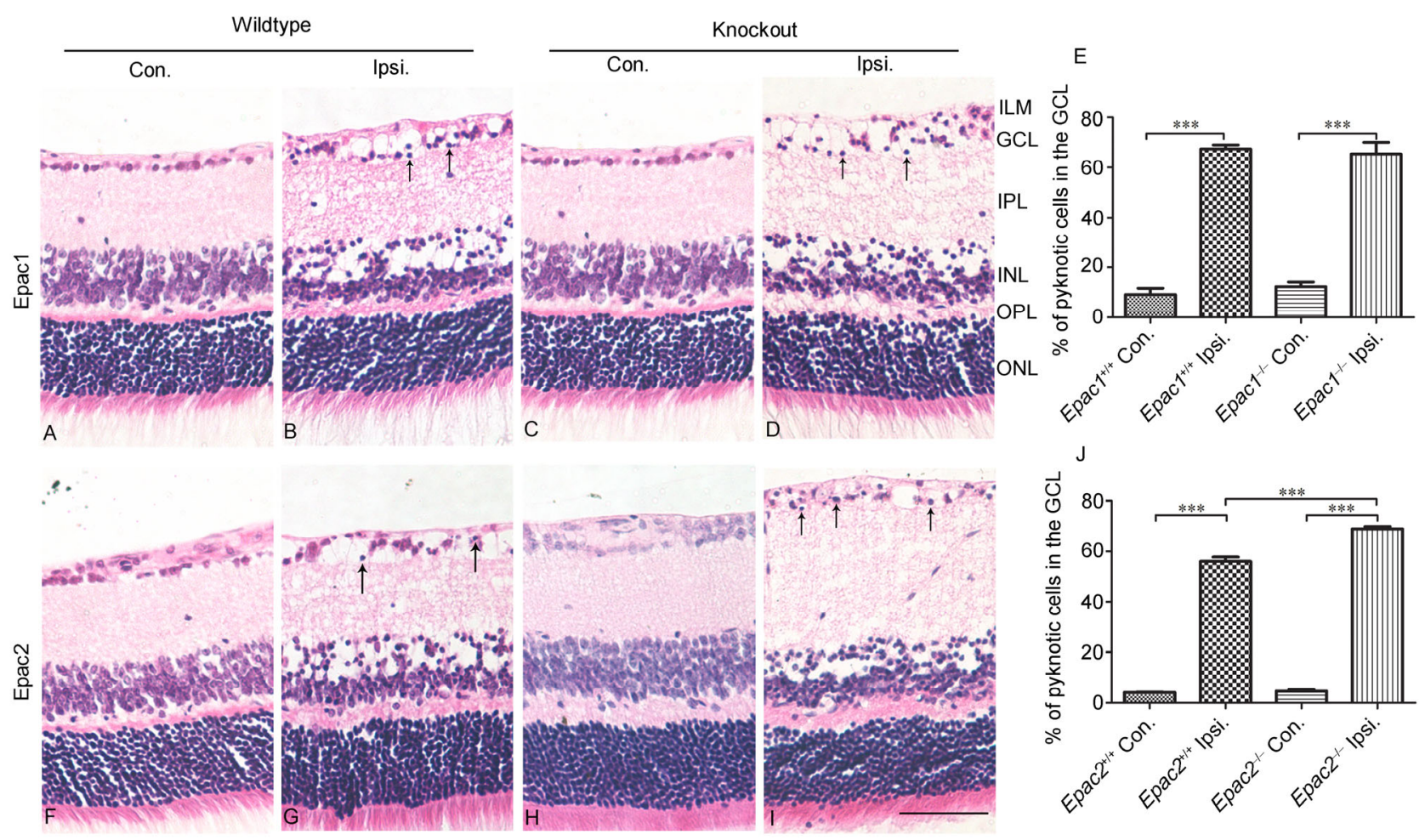

Figure $2 \mathrm{H} \& \mathrm{E}$ staining of retina sections after tMCAO. Microphotographs of $\mathrm{Epacl}^{+/+}$contralateral (A), Epacl ${ }^{+/+}$ipsilateral (B), Epacl ${ }^{-/-}$contralateral (C),

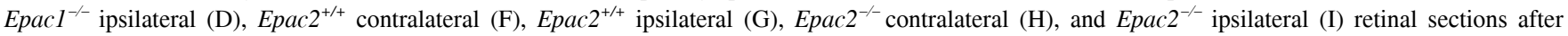
tMCAO were shown. Pyknotic nuclei in GCL were indicated by arrows. Percentage of pyknotic cells in the GCL of Epac1 (E) and Epac2 (J) mice retinae were shown. The photos were taken under $20 \times$ magnification. Scale bar, $50 \mu \mathrm{m} . n=3-6$ in each group; $* * * P<0.001$, two-way ANOVA, followed by Bonferroni post-test. Con., contralateral; Ipsi., ipsilateral; ILM, inner limiting membrane; GCL, ganglion cell layer; IPL, inner plexiform layer; INL, inner nuclear layer; OPL, outer plexiform layer; ONL, outer nuclear layer. 


\subsection{Retinal swelling after tMCAO}

In H\&E-stained sections, the disorganized retina morphology with cell death in GCL and INL layers, and increased IPL thickness of ipsilateral retinae after tMCAO indicated swelling. After tMCAO, slight increase in retinal thickness was observed in the ipsilateral retinae of both $\mathrm{Epacl}^{+/+}$and Epacl $^{-/}$mice, compared with their contralateral retinae. However, no significant difference was found between these two genotypes. In Epac2 $2^{-/}$mice, tMCAO induced an increased retinal thickness in the ipsilateral side, which was mainly due to the increased thickness of IPL. Slightly increased thickness of inner retinal layer was observed in ipsilateral side of $\mathrm{Epac2}^{+/+}$. The inner retinal thickness increase in $\mathrm{Epac}^{-/-}$group was significantly higher than that of ppac2 $^{+/+}$group (Table 1).

Immunocytochemical staining showed that, after tMCAO, the expression of AQP4 was up-regulated in the ipsilateral retinae in all genotypes (Figure $3 \mathrm{~B}, \mathrm{D}, \mathrm{G}$ and I). The ICC scoring indicated up-regulated AQP4 expression after tMCAO in ipsilateral retinae in both $\mathrm{Epacl}^{+/+}$and $\mathrm{Epacl}^{-/-}$ mice, but the extent of AQP4 over-expression was similar in both genotypes (Figure 3B, D and E; effect of tMCAO: $F_{1,16}=105.13, P<0.0001$; effect of genotype: $F_{1,16}=0.13$, $P=0.73$; effect of interaction: $F_{1,16}=0.13, P=0.73$ ). However, compared with contralateral retinae, Epac $2^{-/}$mice showed significantly higher expression of AQP4 in ipsilateral retinae than that of Epac2 ${ }^{+/+}$retinae (Figure 3G, I and J; effect of tMCAO: $F_{1,12}=69.36, P<0.0001$; effect of genotype: $F_{1,12}=8.64, \quad P=0.01$; effect of interaction: $F_{1,12}=11.76$, $P=0.005)$.

\subsection{Retinal glial cell activation after tMCAO}

Glial cell reactivity was demonstrated by immunocytochemical staining for GFAP. Under normal condition, the GFAP signal was restricted in the astrocytes and the end feet of Müller cells, in GCL and OPL respectively (Figure 4A, C, F and H). After tMCAO, activation of Müller cells in the ipsilateral retinae was observed with their processes protruding from the ILM to the outer border of INL (Figure 4B, D, G and I). Ischemia-induced glial cell activation was observed in both $\mathrm{Epacl}^{+/+}$and $\mathrm{Epacl}^{-/-}$ipsilateral retinae, but the extent of glial reactivation in these two genotypes appeared similar (Figure 4E; effect of tMCAO: $F_{1,16}=138.89$, $P<0.0001$; effect of genotype: $F_{1,16}=0.22, P=0.64$; effect of interaction: $\left.F_{1,16}=0.22, P=0.64\right)$. In ipsilateral retinae of Epac2 $^{+/+}$, significant increase of GFAP expression was observed after tMCAO (Figure 4G). In ipsilateral retinae of Epac2 $^{-/-}$mice, significant increase in GFAP expression was also observed when compared with contralateral retinae. However, the extent of increase of GFAP expression in ipsilateral retinae of $\mathrm{Epac}^{-/}$mice was higher than that of Epac2 $^{+/+}$ipsilateral retinae (Figure $4 \mathrm{H}$ and $\mathrm{J}$; effect of tMCAO: $F_{1,12}=101.77, \quad P<0.0001$; effect of genotype: $F_{1,12}=5.77, \quad P=0.03$; effect of interaction: $F_{1,12}=5.77$, $P=0.03)$.

\subsection{Oxidative stress in the retina after tMCAO}

Oxidative stress was determined by staining for Prx6. After tMCAO, contralateral retinae appeared normal morphology (Figure 5A, C, F and H). Compared with contralateral side, more accumulation of Prx6 was observed in the ipsilateral retinae (Figure 5B, D, G and I). The increased Prx6 was observed in ipsilateral side of $\mathrm{Epacl}^{+/+}$and $\mathrm{Epacl}^{-/-}$retinae, but no significant difference was observed in ICC scoring (Figure 5E; effect of tMCAO: $F_{1,16}=18.67, P=0.0005$; effect of genotype: $F_{1,16}=1.52, P=0.23$; effect of interaction: $\left.F_{1,16}=0.38, P=0.55\right)$. ICC scoring showed significant upregulation of Prx6 in Epac2 $2^{-/}$ipsilateral retinae compared with $E p a c 2^{-/}$contralateral retinae, but no significant Prx6 up-regulation was observed in Epac2 ${ }^{+/+}$ipsilateral retinae, and more increase in Prx6 expression was observed in Epac2 $^{-/-}$ipsilateral retinae (Figure 6G, I and J; effect of tMCAO: $\quad F_{1,10}=35.34, \quad P=0.0001 ; \quad$ effect of genotype: $F_{1,10}=7.1, P=0.02$; effect of interaction: $F_{1,10}=7.1, P=0.02$ ).

Table 1 Retinal layer thickness $(\mu \mathrm{m})$ after $\mathrm{tMCAO}^{\mathrm{a}}$

\begin{tabular}{|c|c|c|c|c|c|c|c|}
\hline \multicolumn{2}{|c|}{ Groups of mice } & OLM-ILM & INL & IPL-ILM & INL-ILM & ONL & OPL \\
\hline \multirow{2}{*}{ Epacl $^{+/+}$} & con & $159.2 \pm 14.6$ & $35.6 \pm 3.7$ & $63.1 \pm 5.0$ & $99.3 \pm 9.6$ & $46.5 \pm 3.4$ & $11.0 \pm 0.6$ \\
\hline & ipsi & $167.0 \pm 5.7$ & $35.7 \pm 0.8$ & $74.1 \pm 3.1$ & $107.0 \pm 4.7$ & $47.3 \pm 0.6$ & $10.9 \pm 0.8$ \\
\hline \multirow{2}{*}{ Epac1 $^{-/-}$} & con & $149.0 \pm 8.0$ & $39.0 \pm 4.8$ & $60.1 \pm 3.4$ & $94.8 \pm 6.2$ & $45.6 \pm 1.4$ & $11.4 \pm 0.9$ \\
\hline & ipsi & $154.5 \pm 8.5$ & $36.3 \pm 1.5$ & $64.4 \pm 6.2$ & $98.9 \pm 7.3$ & $48.1 \pm 1.3$ & $10.5 \pm 1.0$ \\
\hline Epac $^{+/+}$ & con & $161.5 \pm 8.8$ & $34.2 \pm 2.3$ & $67.1 \pm 2.3$ & $99.3 \pm 5.3$ & $53.0 \pm 3.6$ & $13.8 \pm 0.5$ \\
\hline \multirow{2}{*}{ Epac2 $^{-/-}$} & con & $163.2 \pm 9.8$ & $38.6 \pm 4.6$ & $62.67 \pm 5.6$ & $99.2 \pm 8.7$ & $53.5 \pm 0.9$ & $13.9 \pm 1.8$ \\
\hline & ipsi & $204.9 \pm 5.5^{*}$ & $41.4 \pm 1.5$ & $96.1 \pm 9.4^{*}$ & $136.0 \pm 7.6^{*}$ & $58.2 \pm 4.3$ & $14.9 \pm 1.0$ \\
\hline
\end{tabular}

a) Data were expressed as mean \pm SEM ( $n=3-6$ in each group). *, $P<0.05$, vs. knockout contralateral side, two-way ANOVA, followed by Bonferroni post-test. OLM: outer limiting membrane; ILM: inner limiting membrane; INL: inner nuclear layer; IPL: inner plexiform layer; ONL: outer nuclear layer; OPL: outer plexiform layer; con: contralateral; ipsi: ipsilateral. 

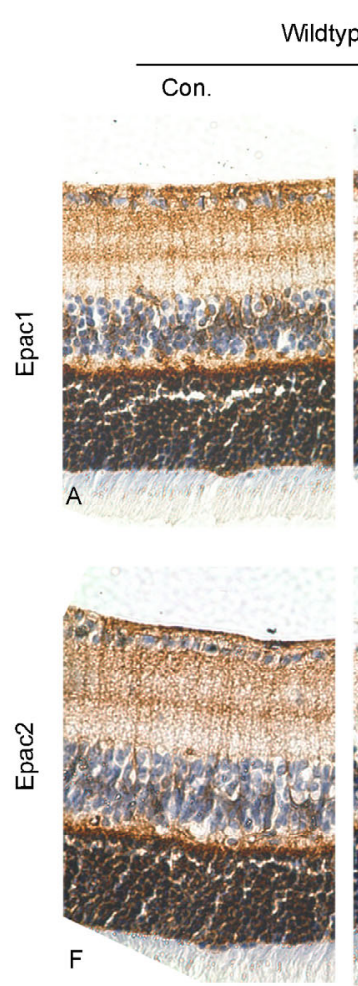
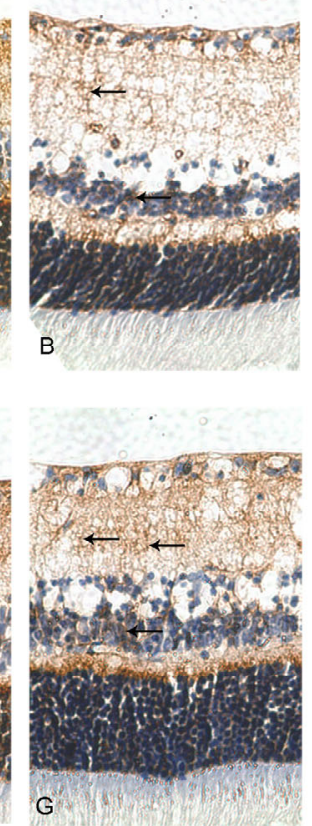
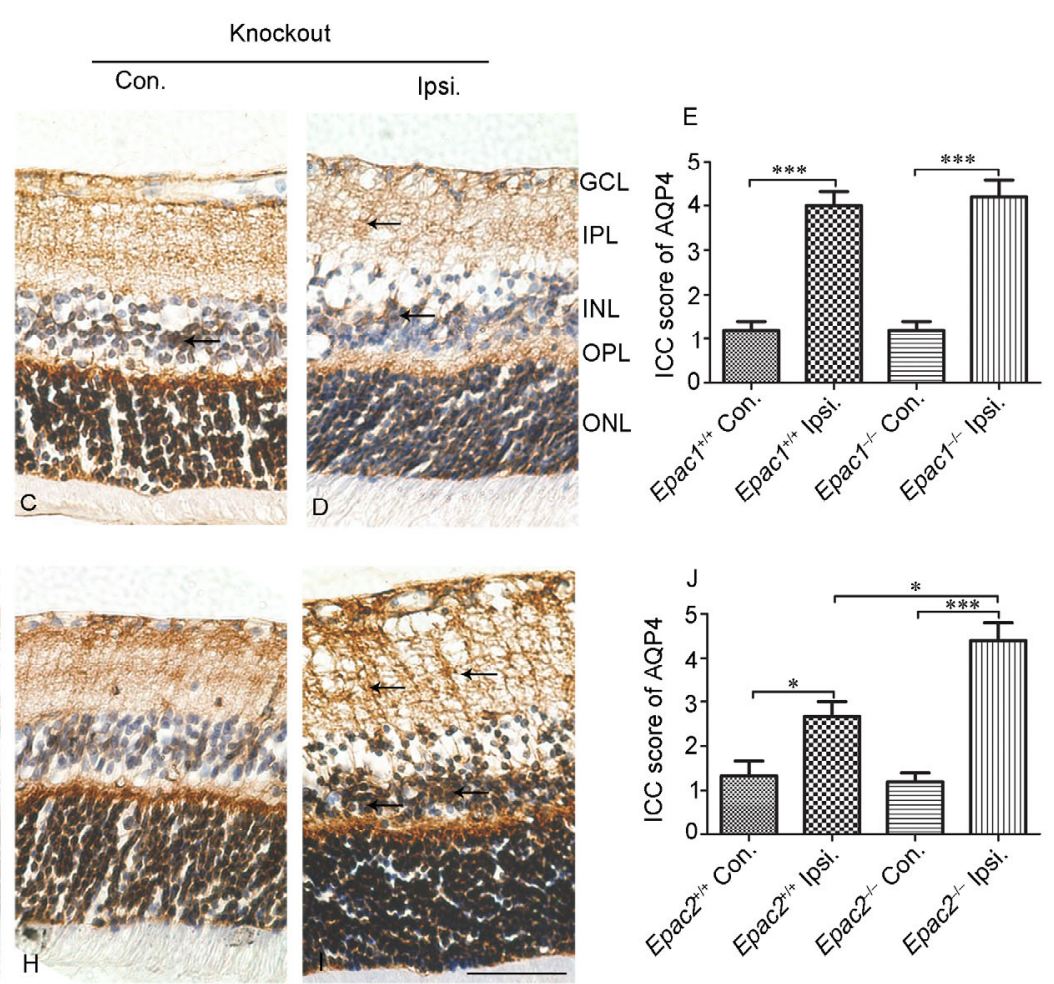

Figure 3 AQP4 immunoreactivity after tMCAO. The representative microphotographs of AQP4 immunoreactivity in $E_{p a c 1^{+/+}}$contralateral (A), Epac1 $^{+/+}$

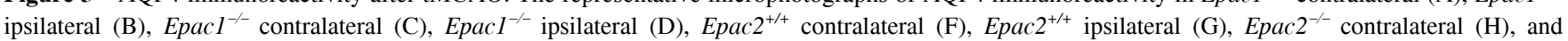
Epac $^{-/}$ipsilateral (I) retinae were shown. Increased expression of AQP4 in the ipsilateral retinae was indicated by arrows. ICC scoring for AQP4 immunoreactivity of Epac1 (E) and Epac2 (J) mice retinae after tMCAO were shown. The $n$ number in each group was 3 to 6 . The micrographs were taken under $40 \times$ magnification. Scale bar, $50 \mu \mathrm{m}$. Semi-quantitative ICC scores were performed, $* P<0.05$; $* * * P<0.001$, two-way ANOVA, followed by Bonferroni post-test. Con., contralateral; Ipsi., ipsilateral; GCL, ganglion cell layer; INL, inner nuclear layer; IPL, inner plexiform layer; ONL, outer nuclear layer; OPL, outer plexiform layer.

\section{Discussion}

The $\mathrm{Epacl}^{-/-}$mice displayed neither developmental nor reproductive abnormalities, but they showed an impaired glucose tolerance and insulin secretion reduction after glucose challenge when compared with their Epacl ${ }^{+/+}$littermates [20]. Epac2 $2^{-/-}$mice also have such insulin secretion dysfunction after glucose stimulation [26]. Under normal condition, the retinae of these knockout mice appear normal. In ischemic retinopathy induced by $\mathrm{tMCAO}$, the retinal neuronal cell loss in GCL is the major damage. Pyknosis is an irreversible condensation of chromatin in the cells undergoing necrosis or apoptosis, which is an indication of cell death. In ischemic retinopathy studies, the pyknotic cells have been used for neuronal cell death measurement $[11,22,23]$. In all mice exposed to tMCAO, the percentage of pyknotic cells in the ipsilateral GCL was significantly higher than that in contralateral retinae. In $\mathrm{Epac}^{-/-}$mice, the ischemia/reperfusion injury resulted in more neuronal cell death, compared with control mice. However, there was no obvious difference between $\mathrm{Epacl}^{+/+}$and $\mathrm{Epacl}^{-/}$ipsilateral side retinae after tMCAO. Our observations suggest that loss of Epac2 may lead to more severe retinal GCL neuronal cell loss after ischemia/reperfusion injury.

Water accumulation occurs after ischemic/reperfusion injury due to the loss of membrane ionic pumps and cell swelling [9]. AQP4 is the main water channel protein that regulates the extracellular and intracellular water exchange bidirectionally. The expression of AQP4 is increased during ischemia, promoting retinal swelling. Glial cell swelling occurs during reperfusion, down-regulating functional potassium channels and reduces transmembrane inward rectifying $\mathrm{K}^{+}$(Kir) currents [27]. Therefore, ischemia/reperfusion process increases extracellular fluid volume and retinal glial cells swelling, resulting in progressive thickening of inner retina, which further contributes to degeneration of photoreceptors and neuronal cells [28]. In Epac2 $2^{-/-}$mice, the ipsilateral retinae showed significant increase in the thickness of inner retina after tMCAO, compared with those of wildtype mice. In the present study, the increased thickness of inner retina was associated with up-regulation of AQP4 after tMCAO. However, the inner retina thickening was not that significant in $\mathrm{Epacl}^{-/}$mice, when compared with $\mathrm{Epacl}^{+/+}$mice after tMCAO.

Glial activation is a well-accepted indicator of retinal injury during stress or diseases conditions, such as glaucoma, hypoxia, ischemia and diabetes $[10,25,29]$. The up-regulated 

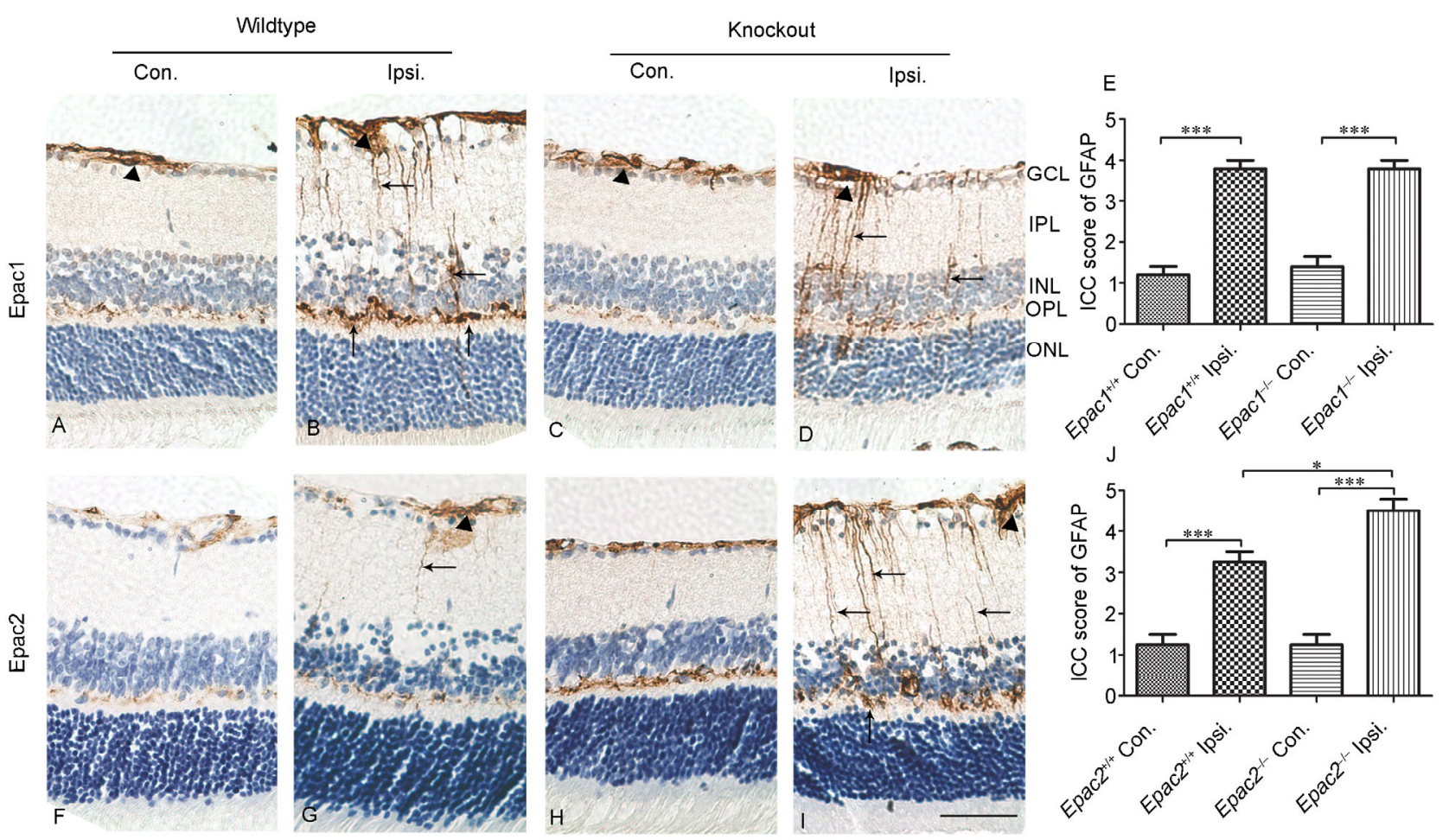

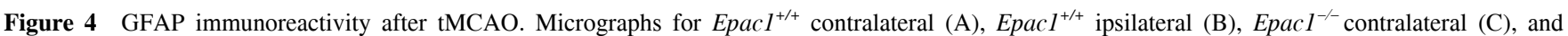

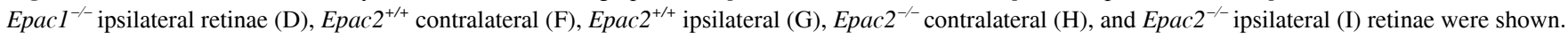
GFAP positive astrocytes (arrowheads) were restricted in GCL. Processes of activated Müller cells (arrows) could be seen in the ipsilateral retinae (B, D, G and I). The $\mathrm{n}$ number in each group was 3 to 6 . The micrographs were taken under $40 \times$ magnification. Scale bar, $50 \mu \mathrm{m}$. Semi-quantitative ICC scores for Epac1 (E) and Epac2 (J) retinae were shown, $* P<0.05$, $* * * P<0.001$, two-way ANOVA followed by Bonferroni post-test. Con., contralateral; Ipsi., ipsilateral; GCL, ganglion cell layer; INL, inner nuclear layer; IPL, inner plexiform layer; ONL, outer nuclear layer; OPL, outer plexiform layer.

immunoactivity of GFAP, a marker of astrocytes and important component of Müller cells, is a well-known sign for glial activation. Under normal conditions, the astrocytes and Müller cells reside in GCL and INL respectively, and the GFAP staining signals are restricted in these two retinal layers. After ischemia/reperfusion insult, the expression of GFAP increased, shown as hypertrophy of astrocytes, and Müller cell processes protruding from GCL to outer ONL. In a 15 months old type 2 diabetes mice model, in which retinal ischemia also occurred, the retinal GFAP expression was higher than that in their non-diabetic littermates [10]. In other studies using tMCAO to induce retinal ischemia/reperfusion injury, GFAP expression is higher in the ipsilateral side retinae compared to their contralateral control retinae $[11,22,25]$. Currently, it is clear that the more intense GFAP staining is an indirect effect due to the more severe ischemic retinopathy induced. Our observations on intense GFAP staining in ipsilateral retinae were consistent with previous data. More GFAP staining was observed in Epac2 $^{-/-}$ipsilateral retinae when compared with Epac2 $^{+/+}$ ipsilateral retinae-the $E p a c 2^{-/-}$retinae suffered more severe retinopathy after tMCAO. In contrast, the difference in GFAP expression in the ipsilateral retinae was not observed in the ipsilateral retinae between $\mathrm{Epacl}^{+/+}$and $\mathrm{Epacl}^{-/-}$ mice.
Ischemia/reperfusion injury is often accompanied with oxidative stress. When ischemia/reperfusion process occurs, the free radicals and proteases are formed which further disrupt brain-cell membranes, causing irreversible damage. Therefore in this study, oxidative stress level was determined to reflect the extent of retinopathy. Prx6 is a member of antioxidant enzyme that belongs to peroxiredoxin family. It functions as a protective factor in ischemia/reperfusion injury [30-32]. Increased expression of Prx6 has been observed in the brain after tMCAO [33]. In our case, increased Prx6 expression in the ipsilateral retinae was observed in parallel with other observations. The extent of the increase of Prx6 was higher in Epac2 $2^{-/-}$mice, but not in other three genotypes.

Epac1 and Epac2 are two isoforms of Epac proteins, with differential expression patterns, which have been reported since the discovery of Epac proteins [18]. Both Epac1 and Epac2 are expressed in the retina [19]. It has been shown that Epacs have a profound role in cognition, loss of Epacs leads to impairment in memory retrieval in a fear conditioning paradigm [34], and it is also noted that in depressed patients, the Epac2 expression but not Epac1 is altered in the cortex [35]. Besides, Epac2 participates in synaptic plasticity $[17,36]$. Such experimental evidence indicates that Epac2 is more involved in CNS. Epac1 protein has been 

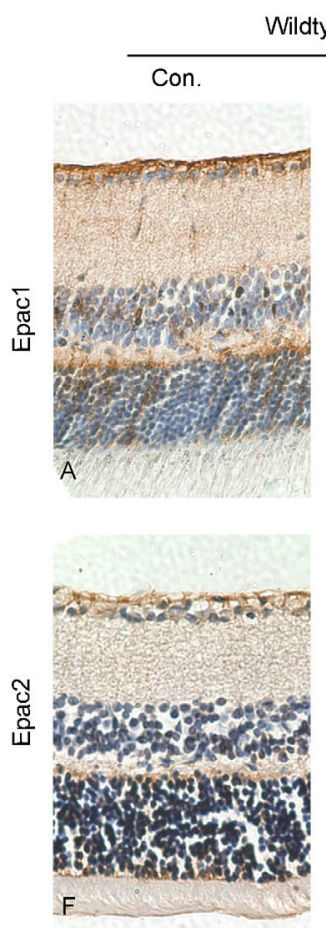

ditype
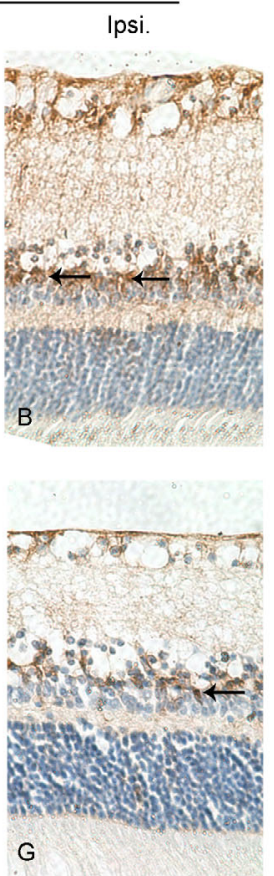
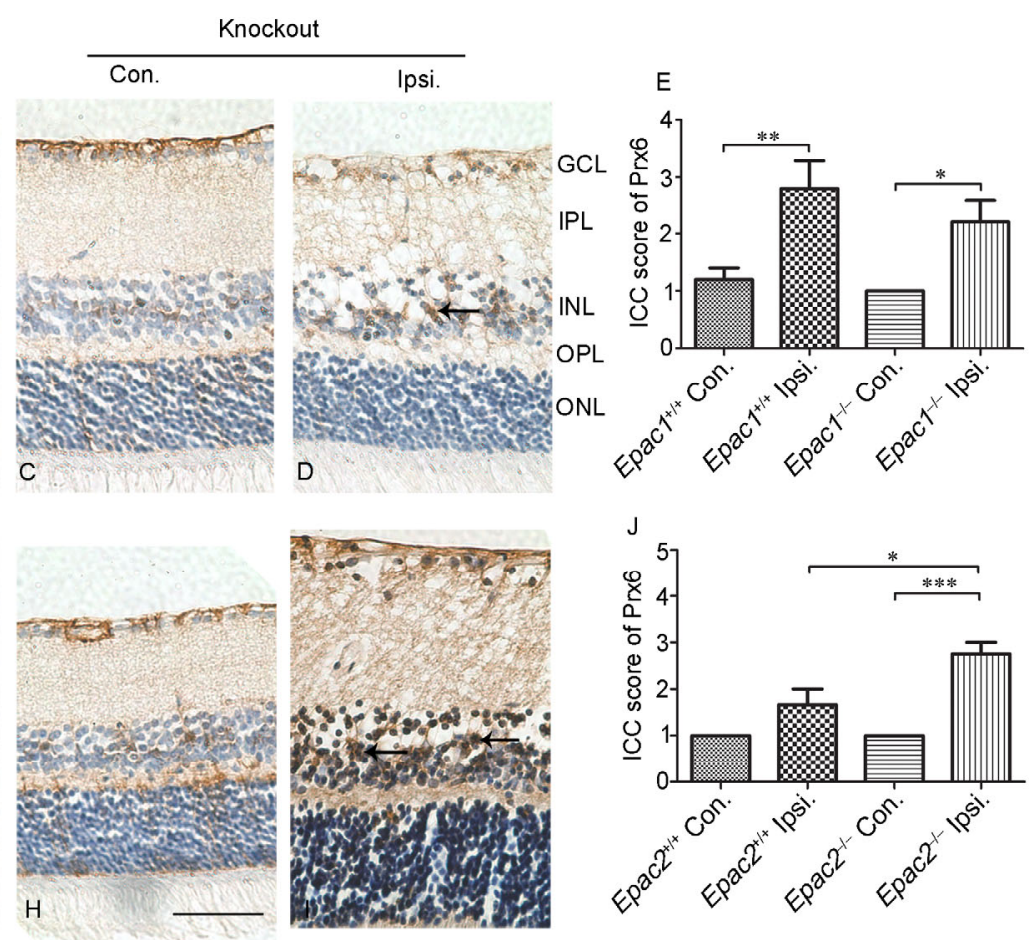

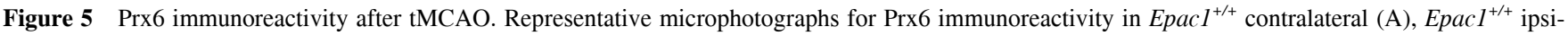

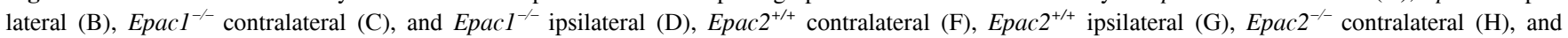
$E_{p a c} 2^{-/}$ipsilateral (I) retinae were shown. Signals from reactive glial cells (arrows) could be seen in the IPL and INL of the ipsilateral retinae. ICC scores of Epac1 (E) and Epac2 (J) mice retinae were shown. Increased expression of Prx6 (arrows) could be seen in the ipsilateral retinae. The $n$ number in each group was 3 to 6 . The micrographs were taken under $40 \times$ magnification. Scale bar, $50 \mu \mathrm{m}$. $* P<0.05$, $* * P<0.01$, $* * * P<0.001$, two-way ANOVA, followed by Bonferroni post-test. Con., contralateral; Ipsi., ipsilateral; GCL, ganglion cell layer; INL, inner nuclear layer; IPL, inner plexiform layer; ONL, outer nuclear layer; OPL, outer plexiform layer.

known for its role in cell-cell contacts and calcium handling in cardiomyocytes [12,37]. Epac1 has been shown to have differential roles in different types of tissues: in cultured cortical neurons, Epac1 promotes cell death after oxidative stress, whereas it protects myocardial cells from oxidative stress injury [38]. The Epac downstream effector, Rap1, has been shown to regulate retinal pigment epithelial cell barrier function [39]. The Epac proteins also participate in the neuronal survival and synaptic plasticity, by increasing $\mathrm{PKB} /$ Akt phosphorylation, in which Epac2 dominates the function, whereas Epac1 functions in a smaller extent [15]. It appears that in CNS, Epac2 plays a beneficial role. Retina is part of central nervous system with complex but well-ordered neuronal circuit organization [40] and can be viewed non-invasively. Therefore, it is reasonable that retinae lacking of Epac2 are more vulnerable to ischemia/reperfusion insult. A recently published paper showed that mice with conditional knockout of both Epac1 and Epac2 (named Epac null) in the forebrain displayed defects in generating artificial long-term potentiation (LTP), whereas Epac1 knockout or Epac2 knockout did not show such phenotype. Besides, only Epac null showed impaired Rap1 activity, neither Epac1 nor Epac2 knockout showed such activity loss in Rap1. These observations indicate that
Epac1 and Epac2 may compensate for each other when either one is not functioning under normal condition [41]. Under extreme conditions, such as ischemia/reperfusion injury, this compensation mechanism might be disrupted. As Epac2 protein is closely related to neuronal functions, in retina, where locate different types of neurons, it is possible that, under certain extreme circumstances, Epac1 may not be able to compensate for the deletion of Epac2, thus Epac2-deficiency leads to more severe damage.

In the present study, we made use of genetically manipulated mice with Epacl or Epac2 gene deletion, combined with $\mathrm{tMCAO}$, to determine the role of Epac proteins in the pathogenesis of ischemic retinopathy. Our data indicated that Epac2 may play an important role in maintaining normal retinal function, since Epac2-deficient mice showed more severe signs of ischemic retinopathy, such as neuronal death, retinal swelling, glial reactivity, and oxidative stress after retinal ischemia/reperfusion. However, the role of Epac1 in pathogenesis of ischemic retinopathy was not as significant as Epac2 based on our observations. Epac1-, Epac2- and Epac1; Epac2-double mutant mice would serve as essential tools to further understand the detailed mechanisms of Epac involvement in the pathogenesis of ischemic retinopathy. 
The authors declare that they have no conflict of interest. All animal experiments in the study were conducted according to the requirements of the Cap. 340 Animals (Control of Experiments) Ordinance and Regulation, and relevant legislation and Codes of Practice in Hong Kong. All the experiments performed were strictly following the experimental guidelines approved by the Committee on the Use of Live Animals for Teaching and Researching (CULATR No. 2239-10).

This work was supported by the Research Grants Council of Hong Kong (RGC) HKU 764008M to Sookja Kim Chung. We thank Professor S. Seino from Kobe University for providing Epac $2^{-/}$mice, and Professor J.L. Bos from Utrecht University for providing Epac antibodies.

1 Osborne NN, Casson RJ, Wood JPM, Chidlow G, Graham M, Melena J. Retinal ischemia: mechanisms of damage and potential therapeutic strategies. Prog Retin Eye Res, 2004, 23: 91-147

2 Buchi ER. Cell death in the rat retina after a pressure-induced ischaemia-reperfusion insult: an electron microscopic study. I. Ganglion cell layer and inner nuclear layer. Exp Eye Res, 1992, 55: 605-613

3 Lafuente MP, Villegas-Pérez MP, Sellés-Navarro I, MayorTorroglosa S, de Imperial JM, Vidal-Sanz M. Retinal ganglion cell death after acute retinal ischemia is an ongoing process whose severity and duration depends on the duration of the insult. Neuroscience, 2002, 109: 157-168

4 Nucci C, Tartaglione R, Rombolà L, Morrone LA, Fazzi E, Bagetta G Neurochemical evidence to implicate elevated glutamate in the mechanisms of high intraocular pressure (IOP)-induced retinal ganglion cell death in rat. Neurotoxicology, 2005, 26: 935-941

5 Yamamoto H, Schmidt-Kastner R, Hamasaki DI, Yamamoto H, Parel JM. Complex neurodegeneration in retina following moderate ischemia induced by bilateral common carotid artery occlusion in Wistar rats. Exp Eye Res, 2006, 82: 767-779

6 Bringmann A, Francke M, Pannicke T, Biedermann B, Faude F, Enzmann V, Wiedemann P, Reichelt W, Reichenbach A. Human Muller glial cells: altered potassium channel activity in proliferative vitreoretinopathy. Invest Ophthalmol Vis Sci, 1999, 40: 3316-3323

7 Kim IB, Kim KY, Joo CK, Lee MY, Oh SJ, Chung JW, Chun MH. Reaction of Muller cells after increased intraocular pressure in the rat retina. Exp Brain Res, 1998, 121: 419-424

8 Da T, Verkman AS. Aquaporin-4 gene disruption in mice protects against impaired retinal function and cell death after ischemia. Invest Ophthalmol Vis Sci, 2004, 45: 4477-4483

9 Yanoff M, Fine BS, Brucker AJ, Eagle Jr. Ralph C. Pathology of human cystoid macular edema. Surv Ophthalmol, 1984, 28(Suppl): 505-511

10 Cheung AK, Fung MK, Lo AC, Lam TT, So KF, Chung SS, Chung SK. Aldose reductase deficiency prevents diabetes-induced bloodretinal barrier breakdown, apoptosis, and glial reactivation in the retina of $\mathrm{db} / \mathrm{db}$ mice. Diabetes, 2005, 54: 3119-3125

11 Cheung AK, Lo AC, So KF, Chung SS, Chung SK. Gene deletion and pharmacological inhibition of aldose reductase protect against retinal ischemic injury. Exp Eye Res, 2007, 85: 608-616

12 Gloerich M, Bos JL. Epac: defining a new mechanism for cAMP action. Annu Rev Pharmacol Toxicol, 2010, 50: 355-375

13 Bos JL. Linking Rap to cell adhesion. Curr Opin Cell Biol, 2005, 17: 123-128

14 Rampersad SN, Ovens JD, Huston E, Umana MB, Wilson LS, Netherton SJ, Lynch MJ, Baillie GS, Houslay MD, Maurice DH. Cyclic AMP phosphodiesterase 4D (PDE4D) Tethers EPAC1 in a vascular endothelial cadherin (VE-Cad)-based signaling complex and controls cAMP-mediated vascular permeability. J Biol Chem, 2010, 285: 33614-33622

15 Nijholt IM, Dolga AM, Ostroveanu A, Luiten PG, Schmidt M, Eisel UL. Neuronal AKAP150 coordinates PKA and Epac-mediated PKB/Akt phosphorylation. Cell Signal, 2008, 20: 1715-1724
16 Penzes P, Woolfrey KM, Srivastava DP. Epac2-mediated dendritic spine remodeling: implications for disease. Mol Cell Neurosci, 2011, 46: $368-380$

17 Woolfrey KM, Srivastava DP, Photowala H, Yamashita M, Barbolina MV, Cahill ME, Xie Z, Jones KA, Quilliam LA, Prakriya M, Penzes P. Epac2 induces synapse remodeling and depression and its disease-associated forms alter spines. Nat Neurosci, 2009, 12: 1275-1284

18 Kawasaki H, Springett GM, Mochizuki N, Toki S, Nakaya M, Matsuda M, Housman DE, Graybiel AM. A family of cAMP-binding proteins that directly activate Rap1. Science, 1998, 282: 2275-2279

19 Whitaker CM, Cooper NGF. Differential distribution of exchange proteins directly activated by cyclic AMP within the adult rat retina. Neuroscience, 2010, 165: 955-967

20 Kai AK, Lam AK, Chen Y, Tai AC, Zhang X, Lai AK, Yeung PK, Tam S, Wang J, Lam KS, Vanhoutte PM, Bos JL, Chung SS, Xu A, Chung SK. Exchange protein activated by cAMP 1 (Epac1)-deficient mice develop beta-cell dysfunction and metabolic syndrome. FASEB J, 2013, 27: 4122-4135

21 Zhang CL, Katoh M, Shibasaki T, Minami K, Sunaga Y, Takahashi $\mathrm{H}$, Yokoi N, Iwasaki M, Miki T, Seino S. The cAMP sensor Epac2 is a direct target of antidiabetic sulfonylurea drugs. Science, 2009, 325: 607-610

22 Cheung SS, Leung JW, Lam AK, Lam KS, Chung SS, Lo AC, Chung SK. Selective over-expression of endothelin-1 in endothelial cells exacerbates inner retinal edema and neuronal death in ischemic retina. PLoS One, 2011, 6: e26184

23 Li SY, Fu ZJ, Ma H, Jang WC, So KF, Wong D, Lo AC. Effect of lutein on retinal neurons and oxidative stress in a model of acute retinal ischemia/reperfusion. Invest Ophthalmol Vis Sci, 2009, 50: 836-843

24 Fu ZJ, Li SY, Kociok N, Wong D, Chung SK, Lo AC. Aldose reductase deficiency reduced vascular changes in neonatal mouse retina in oxygen-induced retinopathy. Invest Ophthalmol Vis Sci, 2012, 53: 5698-5712

25 Li SY, Yang D, Yeung CM, Yu WY, Chang RC, So KF, Wong D, Lo AC. Lycium barbarum polysaccharides reduce neuronal damage, blood-retinal barrier disruption and oxidative stress in retinal ischemia/reperfusion injury. PLoS One, 2011, 6: e16380

26 Shibasaki T, Takahashi H, Miki T, Sunaga Y, Matsumura K, Yamanaka M, Zhang C, Tamamoto A, Satoh T, Miyazaki J, Seino S. Essential role of Epac2/Rap1 signaling in regulation of insulin granule dynamics by cAMP. Proc Natl Acad Sci USA, 2007, 104: 19333-19338

27 Pannicke T, Uckermann O, Iandiev I, Biedermann B, Wiedemann P, Perlman I, Reichenbach A, Bringmann A. Altered membrane physiology in Muller glial cells after transient ischemia of the rat retina. Glia, 2005, 50: 1-11

28 Tso MO. Pathology of cystoid macular edema. Ophthalmology, 1982, 89: 902-915

29 Lo AC, Chen AY, Hung VK, Yaw LP, Fung MK, Ho MC, Tsang MC, Chung SS, Chung SK. Endothelin-1 overexpression leads to further water accumulation and brain edema after middle cerebral artery occlusion via aquaporin 4 expression in astrocytic end-feet. J Cereb Blood Flow Metab, 2005, 25: 998-1011

30 Avellini C, Baccarani U, Trevisan G, Cesaratto L, Vascotto C, D'Aurizio F, Pandolfi M, Adani GL, Tell G. Redox proteomics and immunohistology to study molecular events during ischemiareperfusion in human liver. Transplant Proc, 2007, 39: 1755-1760

31 Eismann T, Huber N, Shin T, Kuboki S, Galloway E, Wyder M, Edwards MJ, Greis KD, Shertzer HG, Fisher AB, Lentsch AB. Peroxiredoxin- 6 protects against mitochondrial dysfunction and liver injury during ischemia-reperfusion in mice. Am J Physiol Gastrointest Liver Physiol, 2009, 296: G266-274

32 Nagy N, Malik G, Fisher AB, Das DK. Targeted disruption of peroxiredoxin 6 gene renders the heart vulnerable to ischemia-reperfusion injury. Am J Physiol Heart Circ Physiol, 2006, 291: H2636-2640

33 Zhang X, Yeung PK, McAlonan GM, Chung SS, Chung SK. Transgenic mice over-expressing endothelial endothelin-1 show cognitive 
deficit with blood-brain barrier breakdown after transient ischemia with long-term reperfusion. Neurobiol Learn Mem, 2013, 101: 46-54

34 Ouyang M, Zhang L, Zhu JJ, Schwede F, Thomas SA. Epac signaling is required for hippocampus-dependent memory retrieval. Proc Natl Acad Sci USA, 2008, 105: 11993-11997

35 Dwivedi Y, Mondal AC, Rizavi HS, Faludi G, Palkovits M, Sarosi A, Conley RR, Pandey GN. Differential and brain region-specific regulation of Rap-1 and Epac in depressed suicide victims. Arch Gen Psychiatry, 2006, 63: 639-648

36 Gekel I, Neher E. Application of an Epac activator enhances neurotransmitter release at excitatory central synapses. J Neurosci, 2008, 28: 7991-8002

37 Roscioni SS, Elzinga CR, Schmidt M. Epac: effectors and biological functions. Naunyn Schmiedebergs Arch Pharmacol, 2008, 377: $345-357$
38 Suzuki S, Yokoyama U, Abe T, Kiyonari H, Yamashita N, Kato Y, Kurotani R, Sato M, Okumura S, Ishikawa Y. Differential roles of Epac in regulating cell death in neuronal and myocardial cells. J Biol Chem, 2010, 285: 24248-24259

39 Wittchen ES, Hartnett ME. The small GTPase Rap1 is a novel regulator of RPE cell barrier function. Invest Ophthalmol Vis Sci, 2011, 52: 7455-7463

40 Kolb $\mathrm{H}$. The architecture of functional neural circuits in the vertebrate retina. The Proctor Lecture. Invest Ophthalmol Vis Sci, 1994, 35: 2385-2404

41 Yang Y, Shu X, Liu D, Shang Y, Wu Y, Pei L, Xu X, Tian Q, Zhang J, Qian K, Wang YX, Petralia RS, Tu W, Zhu LQ, Wang JZ, Lu Y. EPAC null mutation impairs learning and social interactions via aberrant regulation of miR-124 and Zif268 translation. Neuron, 2012, 73: $774-788$

Open Access This article is distributed under the terms of the Creative Commons Attribution License which permits any use, distribution, and reproduction in any medium, provided the original author(s) and source are credited. 